\title{
Design of a new therapy for patients with chronic kidney disease: use of microarrays for selective hemoadsorption of uremic wastes and toxins to improve homeostasis
}

\author{
This article was published in the following Dove Press journal: \\ Drug Design, Development and Therapy \\ 19 January 2015 \\ Number of times this article has been viewed
}

\author{
Mohammad Rashid \\ Shahidi Bonjar' \\ Leyla Shahidi Bonjar² \\ 'School of Dentistry, Kerman \\ University of Medical Sciences, \\ Kerman, Iran; ${ }^{2}$ Department of \\ Pharmacology, College of Pharmacy, \\ Kerman University of Medical \\ Sciences, Kerman, Iran
}

\begin{abstract}
The hypothesis proposed here would provide near to optimum homeostasis for patients with chronic kidney disease (CKD) without the need for hemodialysis. This strategy has not been described previously in the scientific literature. It involves a targeted therapy that may prevent progression of the disease and help to improve the well-being of CKD patients. It proposes a nanotechnological device, ie, a microarray-oriented homeostasis provider (MOHP), to improve homeostasis in CKD patients. MOHP would be an auxiliary kidney aid, and would improve the filtration functions that impaired kidneys cannot perform by their own. MOHP is composed of two main computer-oriented components, ie, a quantitative microarray detector (QMD) and a homeostasis-oriented microarray column (HOMC). QMD detects and HOMC selectively removes defined quantities of uremic wastes, toxins and any other metabolites which is programmed for. The QMD and HOMC would accomplish this with the help of a peristaltic blood pump that would circulate blood aseptically in an extracorporeal closed circuit. During the passage of blood through the QMD, this microarray detector would quantitatively monitor all of the blood compounds that accumulate in the blood of a patient with impaired glomerular filtration, including small-sized, middle-sized and large-sized molecules. The electronic information collected by QMD would be electronically transmitted to the HOMC, which would adjust the molecules to the concentrations they are electronically programmed for and/or receive from QMD. This process of monitoring and removal of waste continues until the programmed homeostasis criteria are reached. Like a conventional kidney machine, MOHP can be used in hospitals and homes under the supervision of a trained technician. The main advantages of this treatment would include improved homeostasis, a reduced likelihood of side effects and of the morbidity resulting from CKD, slower progression of kidney impairment, prevention of end-stage renal failure, a decreased need for hemodialysis therapy, avoidance of dialysis-related side effects later on in the patient's life, improved quality of life and increased life expectancy.
\end{abstract}

Keywords: hemodialysis, uremia, dialysis, homeostasis, microarray, renal failure

\section{Background}

Kidney disease is the eighth most common cause of death worldwide. ${ }^{1}$ Twenty-six million US adults have chronic kidney disease (CKD), ${ }^{2}$ and the global prevalence of the disease is estimated to be $8 \%-16 \%{ }^{3}$ High-risk groups include those with diabetes, hypertension, and a family history of kidney failure. Statistically, up to two-thirds of those affected are patients with diabetes and hypertension. ${ }^{2,4}$ According to one survey, approximately $5 \%-7 \%$ of the world's population has mild CKD, and the disease is more common in developing countries as well as disadvantaged and minority populations. ${ }^{5}$
Correspondence: Leyla Shahidi Bonjar Department of Pharmacology, College of Pharmacy, Kerman University of Medical Sciences, Haft Baagh Blvd, Kerman,

76169I3555, Iran

Tel +989131985712

Fax +98 3432120725

Email shahidileylaa@gmail.com 
CKD, also called chronic kidney failure, refers to the gradual loss of kidney function, ${ }^{6}$ and is manifested by reduced glomerular filtration rate, increased urinary albumin excretion, or both. ${ }^{3,7}$ When the kidneys do not function properly, the homeostatic balance of sodium, potassium, calcium, water, and other salts is impaired. Impaired kidneys lead to accumulation of nitrogenous wastes in the blood and the development of proteinuria, ie, protein in the urine. Uremia develops as nitrogenous wastes accumulate in the blood, and the lack of appropriate water excretion causes edema. Depending on the severity of the disease, the age of the patient, and other factors, CKD manifests as a wide range of physiological disorders during the patient's lifetime. Complications include increased cardiovascular mortality, progression of kidney disease, acute kidney injury, cognitive decline, anemia, bone and mineral disorders, and fractures. ${ }^{3}$ CKD is associated with premature mortality, decreased quality of life, and increased health care expenditure. ${ }^{8,9}$ In addition to diabetes and hypertension, other etiologies of CKD include glomerulonephritis, embryonic malformations, lupus and other diseases that affect the body's immune system, obstruction due to kidney stones, tumors, an enlarged prostate gland in men, repeated urinary tract infections, and polycystic kidney disease. CKD can be assessed using three simple tests, ie, blood pressure, urinary albumin, and serum creatinine. ${ }^{2}$ Persistent proteinuria indicates that $\mathrm{CKD}$ is present. $\mathrm{CKD}$ is differentiated from acute kidney disease by the persistence of decreased kidney function for over 3 months. ${ }^{10-12}$ The sooner CKD is detected, the better the chance of preventing further complications, development of end-stage kidney failure, and a number of life-threatening side effects.

The present work discusses a hypothetical device that could be used to achieve near homeostasis in patients with CKD. The hypothesis aims at developing a microarrayoriented homeostasis provider (MOHP), ie, a computerassisted process of blood filtration that would selectively remove uremic waste and toxins from the blood of a patient with CKD, while circulating blood in an extracorporeal circuit with the aid of a pump. The patient's blood would flow through the tubing and proposed device to get close to constituents of the normal blood plasma near to homeostatic proportion. The device would be preprogrammed to remove set amounts of uremic waste and toxins from the blood, and the settings would be manually adjusted under the direction of a nephrologist according to the needs of the individual patient. The cleansed blood would be return to the body via the circuit. Implementation of this treatment for adjustment of homeostasis would be prescribed by the physician according to the patient's physiological performance. It may be prescribed once or twice a month in a dialysis center; however, some patients may prefer to be treated at home under the supervision of a trained operator.

Figure 1 is a schematic representation of MOHP and its position in the extracorporeal blood circuit. The blood passes through the porous lamellae of a quantitative microarray detector (QMD), which measures the concentrations of uremic waste and toxins of interest. This would be the main electronic role of the QMD. Since the internal environment is dynamic, in an automated progress throughout the entire treatment, in short intervals (eg, every 5 minutes), the QMD resets itself and takes a new score. These electronic QMD readings taken at brief intervals are transmitted continuously to the homeostasisoriented microarray column (HOMC). In this regard, the QMD communicates with the HOMC regarding how much of each compound to retain. HOMC absorbs and immobilizes wastes, as instructed by the QMD. Constant cooperation between the QMD and HOMC is necessary throughout the process to prevent disequilibrium syndrome. When removal is sufficient, the QMD electronically detects the adequacy of hemodialysis or a near homeostasis condition. At this stage, the QMD would signal the end of treatment on the machine monitor, eg, by switching on a green light to attract the attention of the operator, and that would be the end of the treatment session. Throughout treatment, manual samples could be taken from untreated and treated blood to double-check the performance of the device and to determine if any other tests are required. All treated patients should have regular consults with their nephrologist and be evaluated for treatment adequacy, as with conventional hemodialysis. The adequacy assessment should include urea clearance, volume control, blood pressure, mineral metabolism, and clinical symptoms.

\section{General specifications}

MOHP would comprise two main computerized components, ie, the QMD and the HOMC. QMD detects compounds of interest and HOMC immobilizes them. The general specifications of each are discussed as follows. QMD is composed of sets of microarrays residing on porous lamellae that blood can easily pass through. These lamellae are composed of biocompatible polymers on which carbon nanotubes are attached. Because of their surface area, nanotubes provide a large surface area than can harbor an extensive number of specific microarrays. Each set of lamellae and their specific microarrays is designed to detect one specific form of uremic waste (eg, sodium, potassium, calcium, nitrogenous), so a QMD would contain many different types of specialized lamellae. The speed of saturation of each specific microarray 


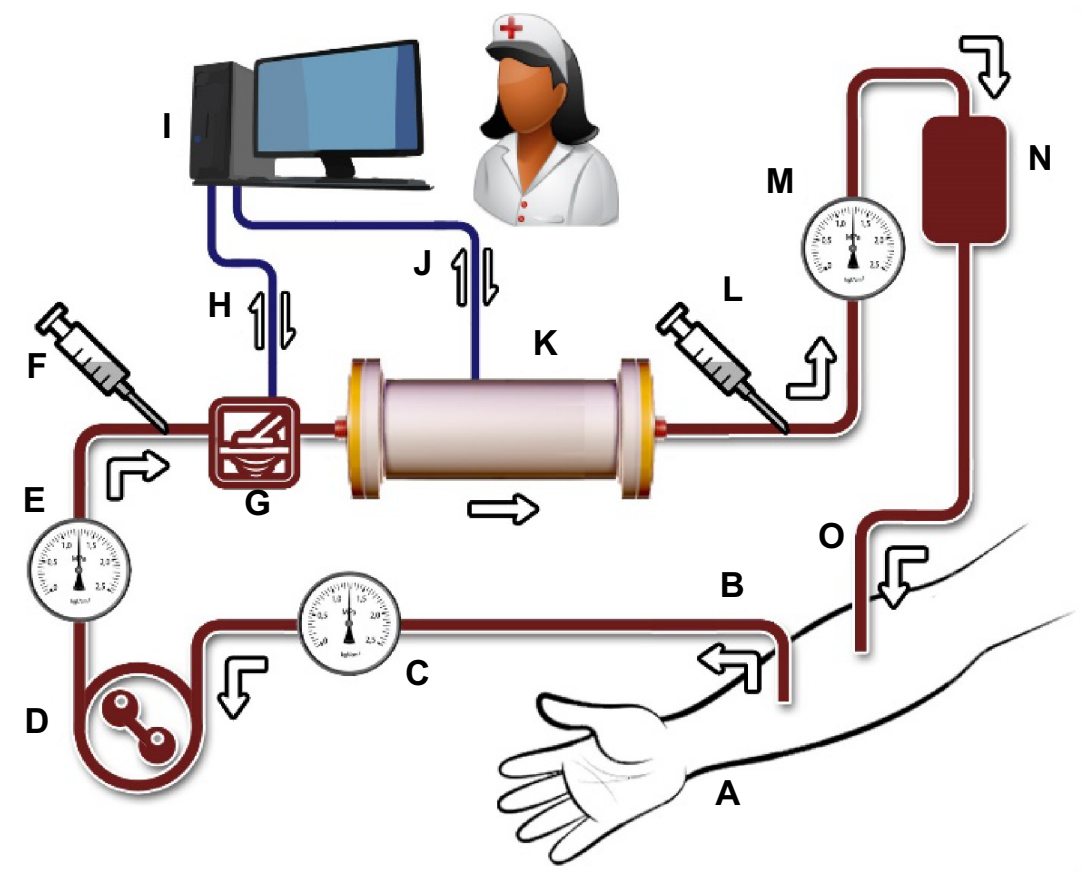

Figure I Microarray-oriented homeostasis provider for selective hemoadsorption of uremic waste and toxins from the blood to improve homeostasis in patients with chronic kidney disease. The device is intended to monitor and adjust the titers of target molecules to near optimum homeostasis. MOHP, with the help of a blood pump, circulates blood aseptically in an extracorporeal closed circuit. The main constituents of the proposed device and treatment would include (A) the patient's hand, (B) blood removed from the artery, (C) an arterial pressure monitor, (D) a blood pump, (E) a heparin pump to prevent blood clotting, (F) a sample of untreated blood, (G) QMD, (H) electronic communication between QMD and COMP, and (I) COMP, including computer hardware, related software, and a trained technician. (J) Electronic communication between COMP and HOMC. (K) HOMC would act as an adsorber cartridge to retain uremic wastes and toxins at levels determined by COMP or as programmed for. As a programmable microarray assembly, HOMC would have adsorptive properties aiming at removing specific ions, molecules, and toxic substances from the patient's blood. Its molecular adherence makes HOMC to target molecules programmed for by COMP. (L) Sampling of treated blood, (M) venous pressure monitor, (N) air detector and air trap, and (O) processed blood returning to the vein. The tubing, QMD, and HOMC are intended for single use and would be appropriately disposed of after each treatment session.

Abbreviations: MOHP, microarray-oriented homeostasis provider; HOMC, homeostasis-oriented microarray column; QMD, quantitative microarray detector; COMP, computer hardware, related software, and a trained technician.

with its target ion or molecule is proportional to the concentration of that material in blood. In other words, the saturation speed induces a magnitude of an electronic pulse that is indicative of the magnitude of concentration of that specific material. These electronic pulses would induce HOMC to remove the wastes accordingly. Given that the concentration of wastes diminishes in the blood and the internal environment is dynamic, new readings are constantly required, so the QMD would be designed to discharge (reset) at short intervals, eg, every 5 minutes. During the discharge process, the ions and molecules retained are released from their retention sites on the QMD, and QMD would start taking new scores to upgrade data information for HOMC performance.

HOMC immobilizes wastes selectively to the concentration level determined by the QMD or to the level manually programmed for by a computer-oriented management panel (Figure 1I). The HOMC would be composed of porous, biocompatible polymeric lamellae, nanotubes, and sets of microarrays similar to those described for the QMD. The difference would be that HOMC confine extensive number of lamellae for irreversible immobilization of waste constituents. Each set of immobilizing lamellae would have the capacity to retain grams of each type of waste. In this regard, quantitatively, HOMC lamellae would be abundant enough not to reach saturation per treatment. Collaboration between QMD and HOMC, under the supervision of a physician, would bring the blood closer and closer to a near homeostasis condition. Achievement of near homeostasis restores the blood close to a normal, healthy balance; however, according to the patient's need, the nephrologist may manually adjust some of the HOMC parameters. The flow rate and total volume of blood being processed at a given point in time would be set by the physician according to the physiological status of the patient. Such tunings would be achievable in computer hardware, related software, and a trained technician of the setup. Figure 1 provides a schematic diagram of the proposed procedure.

\section{Evaluation of the hypothesis}

The efficacy of MOHP should first be evaluated meticulously in large animals, such as dogs, monkeys, or horses, with artificially impaired kidneys that behave functionally like those in human patients with CKD. In short-term comparisons, achievement for near homeostasis should be well demonstrated, along with the well-being of treated animals. 
Clinical signs and symptoms alone are not reliable indicators of treatment adequacy. ${ }^{13}$ The balance of water, sodium, potassium, calcium, removal of nitrogenous wastes, blood pressure, degree of edema, and concentration of hydrogen ions in relation to acidosis should receive special attention at this stage. Long-term evaluations should include lifelong monitoring of the treated animals for progression of kidney impairment, development of end-stage renal disease, weight loss, percent mortality, and average life span. The effectiveness of MOHP in animal investigations would then lead to evaluation in human volunteers with CKD. Researchers in urology should meticulously consider how to enroll and stratify patients according to their profile, especially their history of CKD. Participating patients would be required to sign their informed consent.

Treated patients should be also evaluated for achievement of near homeostasis. When proven to be efficient, MOHP could be used as a technique to assist patients with CKD worldwide. While MOHP would evolve as a new procedure in the treatment of CKD, it would require meticulous scientific attention throughout the evaluation process to ensure that the method is successful. The cost of the device is expected to be similar to that of the hemodialysis machines used in patients with end-stage kidney failure.

\section{Probable discomforts}

Like conventional hemodialysis, MOHP may cause hypotension, muscle cramps, infection, clotting, itching, dry mouth, and anxiety. The cures are the same as suggested by Shahidi Bonjar. ${ }^{14,15}$ Given that the frequency of treatment sessions is much less than for conventional hemodialysis, the probable discomforts are anticipated to be lesser.

\section{Duration of treatment}

Since removal of blood wastes would be a selective process and achievement for near homeostasis would be attained using this target-oriented treatment, it is anticipated that each session of treatment would be performed over a duration of approximately 1-2 hours. Because of its high specificity, it is also anticipated that treatment would be prescribed once or twice a month; however, clinical evaluation under the supervision of skilled researchers and assessment for treatment adequacy would set ideal figures in the future.

\section{Sterilization}

It is anticipated that the tubing and microarray columns would be manufactured and packaged aseptically before leaving the factory. The sterile package would be opened at the time of treatment and administered to the patient with the help of a peristaltic blood pump.

\section{Consequences of the hypothesis and clinical significance}

MOHP is a controlled targeted procedure that determines how much of each of blood components should be removed to attain a near homeostasis condition at each treatment session. Since MOHP is a computerized technique for management of uremic waste and toxins, assessment of treatment adequacy ${ }^{16}$ would reveal efficacy of enhanced physiological criteria in post treatment. However, to ensure near to optimum homeostasis, patients be assessed regularly for treatment adequacy. This assessment should focus mainly on blood pressure, urea clearance, mineral metabolism, volume control, and clinical symptoms. The intelligent electronic behavior of MOHP and its target-oriented nature would help to improve patients' health and enhance their quality of life. It would certainly increase the average life expectancy of patients with CKD and decrease their overall medical expenses in the long term.

\section{Conclusion}

Early detection of kidney disease can help to prevent progression to kidney failure. ${ }^{2}$ The MOHP selectively removes defined quantities of uremic wastes, toxins, and other metabolites from the uremic blood of patients with CKD. This procedure would allow controlled removal of uremic waste and toxins until programmed homeostasis is attained. Its implementation would improve both the quality of life and the life expectancy of CKD patients. In other physiologically similar blood disorders where impaired homeostasis needs correction, similar microarray devices could be developed to help patients.

\section{Acknowledgment}

This paper is dedicated to all patients with CKD and the scientists whose research efforts are focused on improving the health and quality of life of these patients.

\section{Disclosure}

The authors report no conflict of interests in this work.

\section{References}

1. Centers for Disease Control and Prevention. Leading causes of death. Available from: http://www.cdc.gov/nchs/fastats/leading-causes-ofdeath.htm. Accessed July 7, 2014.

2. National Kidney Foundation. About chronic kidney disease. Available from: http://www.kidney.org/kidneydisease/aboutckd.cfm. Accessed July 7, 2014. 
3. Jha V, Garcia-Garcia G, Iseki $\mathrm{K}$, et al. Chronic kidney disease: global dimension and perspectives. Lancet. 2013;382(9888):260-272.

4. Tonelli M, Wiebe N, Culleton B, et al. Chronic kidney disease and mortality risk: a systematic review. J Am Soc Nephrol. 2006;17(7):2034-2047.

5. Couser WG, Remuzzi G, Mendis S, Tonelli M. The contribution of chronic kidney disease to the global burden of major noncommunicable diseases. Kidney Int. 2011;80(12):1258-1270.

6. Mayo Clinic. Diseases and conditions. Chronic kidney disease. Available from: http://www.mayoclinic.org/diseases-conditions/kidneydisease/basics/definition/con-20026778. Accessed July 7, 2014.

7. Wesseling C, Crowe J, Hogstedt C, Jakobsson K, Lucas R, Wegman DH. The epidemic of chronic kidney disease of unknown etiology in Mesoamerica: a call for interdisciplinary research and action. Am J Public Health. 2013;103(11):1927-1930.

8. Centers for Disease Control and Prevention. Prevalence of chronic kidney disease and associated risk factors - United States, 1999-2004. MMWR Morb Mortal Wkly Rep. 2007;56(8):161-165.

9. Levey AS, Atkins R, Coresh J, et al. Chronic kidney disease as a global public health problem: approaches and initiatives - a position statement from kidney disease improving global outcomes. Kidney Int. 2007;72(3):247-259.

10. Levin A, Hemmelgarn B, Culleton B, et al. Guidelines for the management of chronic kidney disease. CMAJ. 2008;179(11):1154-1162.
11. Qaseem A, Hopkins RH, Sweet DE, Starkey M, Shekelle P Screening, monitoring, and treatment of Stage 1 to 3 chronic kidney disease: a clinical practice guideline from the clinical guidelines committee of the American College of Physicians. Ann Intern Med. 2013;159(12):835-847.

12. Levey AS, Coresh J, Balk E, et al. National Kidney Foundation practice guidelines for chronic kidney disease: evaluation, classification, and stratification. Ann Intern Med. 2003;139(2):137-147.

13. National Kidney Foundation. Guidelines for hemodialysis adequacy. Available from: https://www.kidney.org/professionals/kdoqi/ guidelines_updates/doqiuphd_ii.html. Accessed July 7, 2014.

14. Shahidi Bonjar L. Design of a new therapy to treat snake envenomation. Drug Des Devel Ther. 2014;8:819-825.

15. Shahidi Bonjar L. Nanogold detoxifying machine to remove idle nanogold particles from blood stream of cancer patients treated with antibody-nanogold therapeutics. Med Hypotheses. 2013;80:601-605.

16. National Kidney and Urologic Diseases Information Clearinghouse. A service of the National Institute of Diabetes and Digestive and Kidney Diseases, National Institutes of Health. Hemodialysis dose and adequacy. Available from: http://kidney.niddk.nih.gov/kudiseases/pubs/ hemodialysisdose/\#URR. Accessed July 7, 2014.

\section{Publish your work in this journal}

Drug Design, Development and Therapy is an international, peerreviewed open-access journal that spans the spectrum of drug design and development through to clinical applications. Clinical outcomes, patient safety, and programs for the development and effective, safe, and sustained use of medicines are a feature of the journal, which

\section{Dovepress}

has also been accepted for indexing on PubMed Central. The manuscript management system is completely online and includes a very quick and fair peer-review system, which is all easy to use. Visit http://www.dovepress.com/testimonials.php to read real quotes from published authors.

Submit your manuscript here: http://www.dovepress.com/drug-design-development-and-therapy-journal 